“ (C) 2017 IEEE. Personal use of this material is permitted. Permission from IEEE must be obtained for all other uses, in any current or future media, including

reprinting/republishing this material for advertising or promotional purposes, creating new collective works, for resale or redistribution to servers or lists, or reuse of any copyrighted component of this work in other works." 


\title{
Channels Selection using Independent Component Analysis and Scalp Map Projection for EEG-based Driver Fatigue Classification
}

\author{
Rifai Chai, Member, IEEE, Ganesh R. Naik, Senior Member, IEEE, \\ Sai Ho Ling, Senior Member, IEEE, Yvonne Tran, Ashley Craig and \\ Hung T. Nguyen, Senior Member, IEEE
}

\begin{abstract}
This paper presents a classification of driver fatigue with electroencephalography (EEG) channels selection analysis. The system employs independent component analysis (ICA) with scalp map back projection to select the dominant of EEG channels. After channel selection, the features of the selected EEG channels were extracted based on power spectral density (PSD), and then classified using a Bayesian neural network. The results of the ICA decomposition with the backprojected scalp map and a threshold showed that the EEG channels can be reduced from 32 channels into 16 dominants channels involved in fatigue assessment as chosen channels, which included AF3, F3, FC1, FC5, T7, CP5, P3, O1, P4, P8, CP6, T8, FC2, F8, AF4, FP2. The result of fatigue vs. alert classification of the selected $\mathbf{1 6}$ channels yielded a sensitivity of $\mathbf{7 6 . 8 \%}$, specificity of $74.3 \%$ and an accuracy of $75.5 \%$. Also, the classification results of the selected 16 channels are comparable to those using the original 32 channels. So, the selected 16 channels is preferable for ergonomics improvement of EEG-based fatigue classification system.
\end{abstract}

\section{INTRODUCTION}

Driver fatigue is a common problem in transportation which reduces the ability to perform necessary driving skills such as control of the steering, tracking vehicle speed, visual awareness and searching, and sufficient selective attention especially during a monotonous condition of driving for a long period of time. This not only poses a significant risk of injury and fatality to the drivers but also to other road users such as passengers, motorbike users, other drivers and pedestrians. As a result, countermeasures for driver fatigue are needed that are reliable and easy to use in the context of driving $[1,2,3]$.

Countermeasure methods designed to address driver fatigue include those employing: video measurement, psychological assessment and physiological measurement [4]. Video measurement based on facial expression is an indirect measurement of driver fatigue detection while

Rifai Chai, Ganesh R. Naik, Tuan N. Nguyen, Sai Ho Ling, and Hung T. Nguyen are with Centre for Health Technologies, Faculty of Engineering and Information Technology, University of Technology, Sydney (UTS), Broadway NSW 2007, Australia, e-mail: (Rifai.Chai, Ganesh.Naik, Steve.Ling, Hung.Nguyen)@uts.edu.au.

Yvonne Tran is with Centre for Health Technologies University of Technology, Sydney and the Kolling Institute of Medical Research, the University of Sydney (e-mail: Yvonne.Tran@uts.edu.au).

Ashley Craig is with the Kolling Institute of Medical Research, Sydney Medical School, The University of Sydney (e-mail: a.craig@sydney.edu.au) psychological assessment involves frequent self-report of fatigue status using the psychometric questionnaires and this is usually completed off-line. Moreover, a self-report fatigue assessment may result in biased feedback, given it is based on subjective assessment, especially in the context of liability regarding insurance and injury. Physiological measurement of driver fatigue includes using electroencephalography (EEG) to detect brain wave activity [3, 4], electrocardiography (ECG) to detect the heart rate variability [5], electro-oculography (EOG) and other eye tracking system to detect the eye movement [6]. This paper explores countermeasure driver fatigue detection using EEG as it directly measures neurophysiological activity when a person is driving, and is believed to provide a direct assessment of fatigue [4].

To improve the ergonomics of EEG associated with fatigue, fewer EEG channels that provide sensitive detection is clearly preferable, as this will result in greater ease of use and convenience. Independent component analysis (ICA) is a popular technique used for of blind source separation which estimates the source component from the EEG data [7, 8, 9]. Recently, the combination of ICA and feature extraction method has been used for 32 EEG channels driver fatigue classification [3]. To extend this study, current paper explores further use of ICA for selecting the dominant EEG channels for an effective driver fatigue countermeasure system. Here, ICA has the capability to separate EEG signals into independent components related to fatigue activity, and allowing back projection into EEG scalp maps. By further analysing the scalp map projection, the number of dominant EEG channels related to fatigue status can be selected. Therefore, this paper reports the use ICA with scalp map projection to reduce and select dominant EEG channels. A feature extraction algorithm based power spectral density (PSD) and classification algorithm based on Bayesian neural networks are applied to classify fatigue vs. alert states.

\section{Methodology}

\section{A. Block Diagram of Drive Fatigue Analysis}

The method used in this study is shown in Fig.1. The process started with the EEG signal data collection in an experimental condition of simulated driving designed to cause driver fatigue [3]. The channel selection method involved ICA analysis with a projected scalp map and maximum-minimum-threshold criteria to determine the dominant channels. After selecting the EEG channels, EEG features were extracted using PSD and then classified by 
Bayesian neural network.

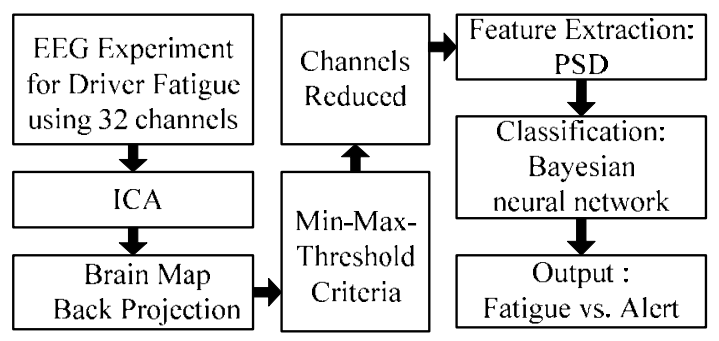

Figure 1. Block diagram of driver fatigue analysis in this study

\section{B. Data Collection of Drive Fatigue Experiment}

The EEG dataset was obtained from previous experimental studies [4]. Institutional Human Research Ethics Committee approval was obtained to conduct this study with 43 healthy participants aged between 18 and 55 years. The study used a driving simulator called Divided Attention Steering Simulator (DASS, Stowood Scientific Instruments). Participants performed a monotonous simulated driving task for a maximum of 2 hours of simulated driving. During the simulation task, participants were required to maintain driving at the centre of the road. EEG, EOG, video recording of facial activity and subjective self-reported fatigue were taken. Also a reaction time response to a target on the computer screen was measured, the target appearing at random times during the simulated driving at any four corners of the screen.

To verify the occurrence of fatigue the following strategies were used: (i) EOG analysis of eye blink rate and eye closure; (ii) reaction time of the divided attention task; (iii) subjective self-reported fatigue using scientifically validated tools such as the Chalder Fatigue Scale and the Stanford Sleepness Scale [4].

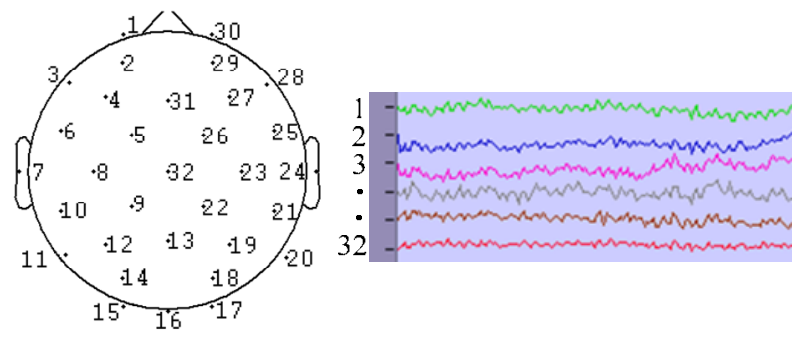

Figure 2. EEG Experiment with its location of 32 EEG Channels

During the experiment, EEG was assessed in 32 channels using the Active-Two EEG system (Biosemi) with the electrode positions as shown in fig.2 which are: FP1(1), AF3(2), F7(3), F3(4), FC1(5), FC5(6), T7(7), C3(8), CP1(9), CP5(10), P7(11), P3(12), PZ(13), PO3(14), O1(15), OZ(16), O2(17), PO4(18), P4(19), P8(20), CP6(21), CP2(22), C4(23), T8(24), FC6(25), FC2(26), F4(27), F8(28), AF4(29), FP2(30), FZ(31) and CZ(32) [4].

The data was divided into alert and fatigue data. The alert data was taken from the first five minutes of the EEG dataset at the beginning of the experiment. Fatigue data was taken from the last five minutes of the EEG dataset where fatigue was identified and verified. The alert and fatigue data were divided into segments of 20s and the first 20s segments with the least movement artifact were used for further analysis.

\section{Independent Component Analysis (ICA) with the Brain Map Back Projection and Min-Max-Threshold Criteria}

ICA performs a blind source separation (BSS) of the EEG data $(x)$ based only on the criterion of the resulting source time courses which are maximally independent. In this study EEGLAB was used to perform ICA [7, 9]. In ICA, the recovered source signal $u$ can be found by applying an unmixing matrix $W(m \times n)$ to the EEG data $x$, as follows:

$$
u=W x \quad x=W^{-1} u
$$

where $W$ denotes the unmixing matrix as the spatial filter for independence component (IC) estimation, $W^{1}$ (inverse of $W$ ), is the $n \times n$ mixing matrix with the columns has the relative weights of back-projected components to each of the scalp channels. The portion of the original EEG data $x$ forms the $i$-th IC $\left(x_{\mathrm{i}}\right)$ which is the product of the two vectors; the $i$ th column of $W$ and the $i$-th row of $u$, and the whole EEG data is the sum of the back-projected ICs of $x_{\mathrm{i}}$, as follows:

$$
x_{i}=W_{i}^{-1} u_{i} \quad x=\sum x_{i}
$$

A visualization of scalp map back-projection of each component can be generated by mapping weights of $W^{1}$ to the corresponding EEG channels on the head model, as the back-projected components into the scalp. Next, the highest and the lowest values in the weights of each component were selected together with a min-max-threshold criteria to indicate significant features from particular channels and resulted in a channels selection with reduced channels.

\section{Feature Extraction and Classification}

Before feature extraction, data from the selected channels were segmented by applying a moving window of $2 \mathrm{~s}$. For the feature extraction, the power spectral density (PSD) was applied to $2 \mathrm{~s}$ ( 512 points for $256 \mathrm{~Hz}$ of sampling rate) to each EEG segment $[3,10]$. This converts the time domain of EEG data segment into frequency domain. EEG bands used for the features include delta $(0.5 \mathrm{~Hz}-3 \mathrm{~Hz})$, theta $(3.5 \mathrm{~Hz}-$ $7.5 \mathrm{~Hz})$, alpha $(8 \mathrm{~Hz}-13 \mathrm{~Hz})$ and beta $(13.5 \mathrm{~Hz}-30 \mathrm{~Hz})$. The total PSD of each EEG band was calculated using the numerical integration of trapezoidal rule, and this provided 4 units of power values per channels, resulting in $4 \times n$ channel units.

The Bayesian neural network was used as a classification algorithm which was able to handle the overfitting issue for the non-linear classification of biomedical signals [11]. The probability distribution of the network parameters is considered in Bayesian learning to provide the best generalization of the network. The Bayesian neural network 
structure uses a three layered feed-forward structure and modeled by:

$$
z_{k}(x, w)=f\left(b_{k}+\sum_{j=1}^{l} w_{k j} f\left(b_{j}+\sum_{i=1}^{m} w_{j i} x_{i}\right)\right)
$$

where $f($.) is the transfer functions ( in this case hyperbolic tangent function), $m$ is the input nodes number $(i=1,2, \ldots$, $m), l$ is the hidden nodes number $(j=1,2, \ldots, l), p$ is the number of output $(k=1,2, \ldots, p), w_{j i}$ is the weight to the hidden unit $y_{j}$ from input unit $x_{i}, w_{k j}$ denotes the weights to output $\left(z_{k}\right)$ from hidden unit $\left(y_{j}\right), b_{j}$ and $b_{k}$ are the biases. According to Bayesian framework [12], the performances function is calculated as follows:

$$
F(w)=\beta E_{D}(w)+\alpha E_{W}(w)
$$

where $\alpha$ and $\beta$ are hyper-parameters with the ratio $\alpha / \beta$ that controls the effective complexity of the network structure, $E_{D}(w)$ is the error function, and $E_{W}(w)$ is the sum square of weight function. By introducing hyper-parameters in the cost function, the weights of neural network can be prevented from being too large, which would result in poor generalization for new test cases. As a result, a validation set is not required in a neural network training procedure. The optimal network structure is the network that has the highest $\log$ evidence value. For the performance indicators of the classification, the sensitivity (true positive rate), specificity (true negative rate) and accuracy were reported.

\section{RESULTS}

ICA was applied using EEGLAB, based on an Infomax ICA algorithm [7]. The dataset for the ICA analysis was constructed in $n \times t \times p$ dimension of data, where, $n \times t$ represents each EEG dataset ( $n$-channels; in this study 32 channels, $t$ represents 20 seconds duration of the EEG data) and $p$ represents number of participant (in this study $p$ equals 43 participants. The ICA analysis returns 32 weighted (overall ICA results for 43 participants) source components. From the result of the ICA, an inverse weight matrix $W^{1}$ was obtained which represents the spatial distribution of each source component on the original 32 EEG channels. By mapping back these weights to 32 channels on the scalp, an ICA scalp map back projection can be obtained as shown in Fig3. The weight value in each component has different peaks with values between -4.01 and +5.27 units of amplitude and this is visualized in different colors in the scalp map (red and blue the highest value of amplitude but in opposite polarity).

By finding the maximum and minimum values for each column of the inverse weight matrix $W^{1}$, a represented channel can be found. A threshold value was applied with a value of $70 \%$ of more than or less than the maximum inverse weight matrix value for the final channel selection, with the result shown in Table 1. The result shows that there were 14
EEG channels that provided the maximum weight value above +3.75 including: P8(20), O1(15), T8(24), P4(19), P3(12), CP6(21), T7(7), CP5(10), F3(4), FC1(5), FC2(26), $\mathrm{AF} 4(29), \mathrm{FC} 5(6), \mathrm{AF} 3(2)$ and $2 \mathrm{EEG}$ channels that provided minimum value lower than +3.75 including F8(28) and FP2(30)). This provided a selection of 16 EEG channels as the chosen channels, from the lower to higher electrode number which includes: AF3, F3, FC1, FC5, T7, CP5, P3, O1, P4, P8, CP6, T8, FC2, F8, AF4 and FP2.

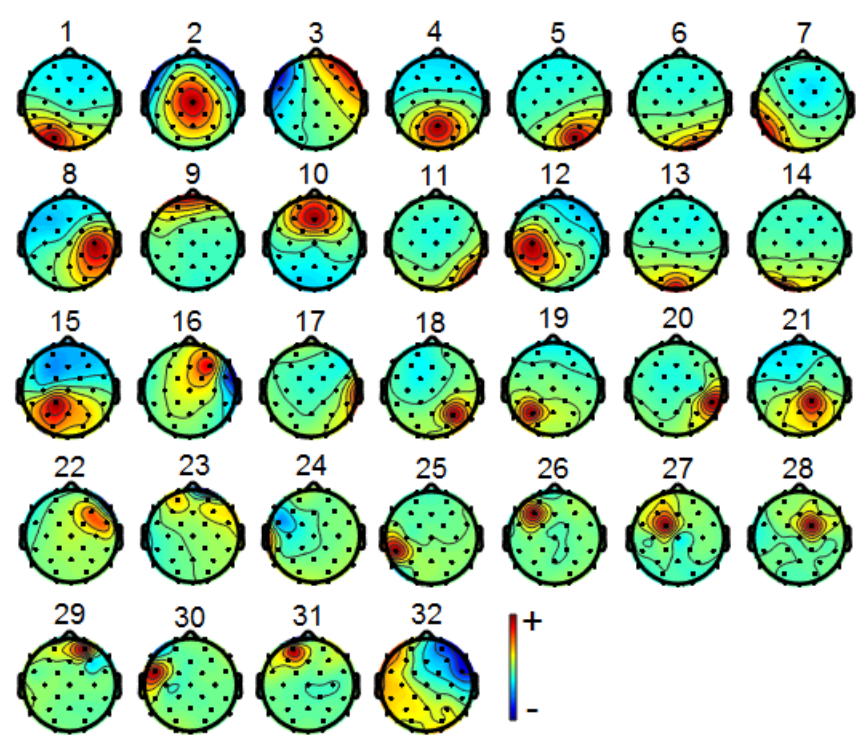

Figure 3. Scalp map of the dominant features for fatigue driver

TABLE I. THE SELECTED EEG CHANELS AFTER APPLYING THE MINMAX AND THRESHOLD

\begin{tabular}{|c|c|c|}
\hline \hline ICA_Component & Channels Name (Number) & Weight Value \\
\hline Value > 70\% of the maximum inverse weight matrix $W^{-1}$ \\
\hline 11 & P8(20) & +4.25 \\
\hline 14 & O1(15) & +4.15 \\
\hline 17 & T8(24) & +4.46 \\
\hline 18 & P4(19) & +4.31 \\
\hline 19 & P3(12) & +4.35 \\
\hline 20 & CP6(21) & +4.44 \\
\hline 24 & T7(7) & +4.40 \\
\hline 25 & CP5(10) & +5.08 \\
\hline 26 & F3(4) & +5.27 \\
\hline 27 & FC1(5) & +4.44 \\
\hline 28 & FC2(26) & +4.99 \\
\hline 29 & AF4(29) & +4.92 \\
\hline 30 & FC5(6) & +4.70 \\
\hline 31 & AF3(2) & +4.14 \\
\hline 22 & F8(28) & -4.01 \\
\hline Value $<70 \%$ of the minimum inverse weight matrix $W^{1}$ \\
\hline 23 & FP2(30) & \\
\hline & & \\
\hline
\end{tabular}

A classification between alert state and fatigue state was done based on the 16 EEG channels. For comparison, the result of the classification using the remaining of unchosen $16 \mathrm{EEG}$ channels is also provided. In addition, the classification using the original 32 EEG channels is provided. Before feature extraction, data from the selected channels were segmented by applying a moving window of 
$2 \mathrm{~s}$ with overlapping $1.75 \mathrm{~s}$ to the $20 \mathrm{~s}$ segments of fatigue and alert data. This provided 73 overlapping segments from one participant. As a result, 3139 units for alert state and 3139 units for fatigue state from the 43 participants were obtained. The PSD as a frequency domain algorithm was used for the feature extraction algorithm. The total power of the 4 EEG bands (delta, theta, alpha and beta bands) was calculated, resulting 4 units of total PSD value from each EEG channels, that is, 64 units of total PSD from the 16 dominant EEG channels.

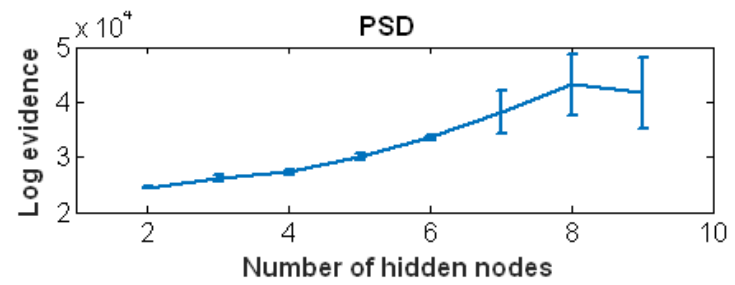

Figure 4. Log evidence from the Bayesian neural network classifier training for using the dominant 16 EEG channels

For the training of the Bayesian neural network, the dataset was divided into $50 \%$ for the training set and the remaining $50 \%$ used for the test set. Table II, shows the classification result with the classification algorithm based on the Bayesian neural network. The plot of the log evidence against the optimum number of hidden nodes of the Bayesian neural network classifier is shown in Fig 4. The optimum number of hidden nodes was 8 to achieve the highest classification of the selected 16 EEG channels for classification fatigue vs. alert states using the dominant 16 EEG channels.

TABLE II. THE RESULS OF CLASSIFICATION

\begin{tabular}{|c|c|c|c|}
\hline \hline Channels used & Sensitivity & Specificity & Accuracy \\
\hline The dominant 16 EEG channels & $76.8 . \%$ & $74.3 \%$ & $75.5 \%$ \\
\hline The least dominant 16 EEG channels & $71.6 \%$ & $61.2 \%$ & $66.4 \%$ \\
\hline The original 32 EEG channels & $77.2 \%$ & $75.6 \%$ & $76.4 \%$ \\
\hline \hline
\end{tabular}

The result of classification on the test sets shows that using the dominant $16 \mathrm{EEG}$ channels resulted in a sensitivity of $76.8 \%$, a specificity of $74.3 \%$ and an accuracy of $75.5 \%$. For comparison, the result using the rest of un-selected 16 EEG channel resulted in a lower sensitivity of $71.6 \%$, specificity of $61.2 \%$ and accuracy of $66.4 \%$. The classification using 32 EEG channels resulted in slightly higher values of sensitivity at $77.2 \%$, specificity at $75.6 \%$ and accuracy at $76.4 \%$.

Given that the 32-channels provide minimal benefit, the dominant 16 EEG channels selected by ICA is preferable, given it involves $50 \%$ fewer channels with an overall accuracy of less than $1 \%$. This suggest the selected 16 channels based on the ICA with its scalp map back projection and the min-max-threshold of the inverse weight matrix approaches, results in an acceptable sensitivity, specificity and accuracy.

\section{CONCLUSION}

In this study, a channels selection method has been applied based on ICA with a back projected scalp map and min-max-threshold method of the inverse weight matrix of the ICA. The result of the channels selection process resulted in 16 EEG channels being selected, including $\mathrm{AF} 3$, F3, FC1, FC5, T7, CP5, P3, O1, P4, P8, CP6, T8, FC2, F8, $\mathrm{AF} 4, \mathrm{FP} 2$ from the original 32-EEG channels. The feature extractor based on PSD and the classifier based on Bayesian neural network were applied to these 16 EEG channels to classify the alert and fatigue states. The classification results based on the dominant 16 EEG channels provided a sensitivity of $76.6 \%$, a specificity of $74.3 \%$ and an accuracy of $75.5 \%$. For comparison, the classification using the least dominant 16 EEG channels resulted in a lower sensitivity, specificity and accuracy. Also the overall accuracy using the selected 16 EEG channels was comparable in sensitivity, specificity and accuracy to a classification using the $32 \mathrm{EEG}$ channels. This suggests that the implementation of an ICA technique with back projected scalp map and min-maxthreshold method is able to select the dominant EEG channels, and therefore holds great promise for developing effective EEG-based fatigue countermeasures.

\section{REFERENCES}

[1] P. A. Desmond, M. C. Neubauer, G. Matthews, and P. A. Hancock, The Handbook of Operator Fatigue: Ashgate Publishing, Ltd., 2012.

[2] S. K. Lal, A. Craig, P. Boord, L. Kirkup, and H. Nguyen, "Development of an algorithm for an EEG-based driver fatigue countermeasure," $J$. Safety Res., vol. 34, pp. 321-328, 2003.

[3] R. Chai, G. Naik, T. Nguyen, S. Ling, Y. Tran, A. Craig, and H. T. Nguyen, "Driver Fatigue Classification with Independent Component by Entropy Rate Bound Minimization Analysis in an EEG-based System," IEEE Journal of Biomedical and Health Informatics, 2016 (In Press).

[4] A. Craig, Y. Tran, N. Wijesuriya, and H. Nguyen, "Regional brain wave activity changes associated with fatigue," Psychophysiology, vol. 49, pp. 574-582, 2012.

[5] Y. Tran, N. Wijesuriya, M. Tarvainen, P. Karjalainen, and A. Craig, "The relationship between spectral changes in heart rate variability and fatigue," J. Psychophysiology, vol. 23, pp. 143-151, 2009.

[6] S. Hu and G. Zheng, "Driver drowsiness detection with eyelid related parameters by Support Vector Machine," Expert Systems with Applications, vol. 36, pp. 7651-7658, 2009.

[7] A. Delorme and S. Makeig, "EEGLAB: an open source toolbox for analysis of single-trial EEG dynamics including independent component analysis," J. Neurosci. Methods, vol. 134, pp. 9-21, 2004.

[8] Y. Tran, A. Craig, P. Boord, and D. Craig, "Using independent component analysis to remove artifact from electroencephalographic measured during stuttered speech," Medical and Biological Engineering and Computing, vol. 42, pp. 627-633, 2004.

[9] J. L. Chen, T. Ros, and J. H. Gruzelier, "Dynamic changes of ICA-derived EEG functional connectivity in the resting state," Human brain mapping, vol. 34, pp. 852-868, 2013.

[10] L. J. Trejo, K. Kubitz, R. Rosipal, R. L. Kochavi, and L. D. Montgomery, "EEG-Based Estimation and Classification of Mental Fatigue," Psychology, vol. 6, p. 572, 2015.

[11] H. T. Nguyen, "Intelligent technologies for real-time biomedical engineering applications," Int. J. Autom. Control, vol. 2, Nos. 2/3, pp. 274-285, 2008.

[12] H. B. Demuth, M. H. Beale, O. De Jess, and M. T. Hagan, "Neural network design," 2014. 